\title{
An Agent-based Collective Model to Simulate Peer Pressure Effect on Energy Consumption
}

\author{
Fatima Abdallah, Shadi Basurra, and Mohamed Medhat Gaber \\ School of Computing and Digital Technology, Birmingham City University \\ \{fatima.abdallah, shadi.basurra, mohamed.gaber\}@bcu.ac.uk
}

\begin{abstract}
This paper presents a novel model for simulating peer pressure effect on energy awareness and consumption of families. The model is built on two well-established theories of human behaviour to obtain realistic peer effect: the collective behaviour theory and the theory of cognitive dissonance. These theories are implemented in a collective agentbased model that produces fine-grained behaviour and consumption data based on social parameters. The model enables the application of different energy efficiency interventions which aim to obtain more aware occupants and achieve more energy saving. The presented experiments show that the implemented model reflects the human behaviour theories. They also provide examples of how the model can be used as an analytical tool to interpret the effect of energy interventions in the given social parameters and decide the optimal intervention needed in different cases.
\end{abstract}

\section{Introduction}

Increased energy consumption generated from fossil fuels is causing high carbon emissions and increased global temperature which is mainly attributed to human actions rather than nature [1]. A significant part of the human effect is accounted for the residential sector which consumes high percentages of the world's electricity consumption (23-31\%) [2]. Although many technological and structural improvements are suggested to decrease energy consumption, occupants' behaviour plays an important role in this matter [3]. A human solution is based on peer pressure, knowing that human actions are mostly affected by the behaviour of others [4]. Hence, it is suggested that policy makers work on stimulating peer pressure to encourage energy efficient behaviour.

This paper presents an Agent-Based Model (ABM) that studies the collective peer pressure effect on energy consumption in a family environment (hereafter family pressure). The occupant agent's peer effect behaviour is inspired by two theories of human social behaviour: collective behaviour by Granovetter [5] and cognitive dissonance by Festinger [6]. The model then adds two types of interventions that aim to enhance the occupants' energy awareness and thus reduce their consumption. The presented model offers a tool that enables analysing the outcomes of energy efficiency interventions in different social conditions. The paper is organised as follows. The next section presents related work including similar ABMs. The used human behaviour theories and available energy interventions 
are presented in Section 3.1. Section 4 presents the ABM that simulates family pressure and energy efficiency interventions, and explains how the behaviour theories were adapted to the application at hand. Section 5 presents the results of simulating a number of scenarios showing how the model can be used to determine the efficiency of interventions in these scenarios. Finally, Section 6 concludes the paper with a summary and pointers for future directions.

\section{Related Work}

Agent-based modelling is considered the most suitable technique to simulate social interaction [7]. An agent-based model is composed of a group of autonomous software components, called agents, which take decisions based on their state and rules of behaviour. The collective agents' decisions cause changes in the environment which is observed and analysed [8]. The technique has been widely used to study occupants energy consumption behaviour.

Among existing ABMs, there are few that simulate occupants' behaviour change due to peer effect. Azar and Menassa [9] propose a model that adds occupants' energy consumption characteristics and interaction to traditional energy simulation tools. The peer effect model is based on the level of influence of individuals and the number of occupants in each level of consumption. However, the used behaviour change model is not theoretically grounded. Models that involve human behaviour simulations need to be validated using huge amounts of real data, and if not available, need to be based on well established and accepted human behaviour theories. Another ABM that simulates social interactions is Chen et al. [10] who explore the effect of peer network structures on the energy consumption in a residential community. The occupant agents decrease their consumption when the consumption of connected occupants is less than that of the agent. On the other hand increasing the agent's consumption is based on a constant probability that represents the percentage of occupants who increase their consumption with no effect from peers. However, it is more logical that peer effect happens in both directions so that high energy consumers may affect others and cause them to increase their consumption in the same way low energy consumers may affect others. Network structures were also studied in Azar and Menassa [11] which is applied in an office environment. The model uses the relative agreement theory which is applied in a community of heterogeneous culture and values. Thus, behaviour change starts between close individuals. However, in a family environment, which is the case in the current paper, it is common that family members have similar culture and values. Therefore, other behaviour change theories need to be applied which will be detailed in Section 3.1.

Studies in [10] and [11] vary the structure of peer networks based on the fact that not all individuals in a community are connected. While in a family environment, family members are always connected at least at night. Therefore, in the current model, the agents are structured in a fully connected network. Another difference between the currently proposed model and existing models [9$11]$ is related to the occupant awareness modelling. Existing models characterise occupants by one attribute which is the average yearly/monthly consumption. This attribute does not only reflect the awareness of occupants, but also the 
time they spend in the building. Hence, it is hard to distinguish if high energy consumption is due to low awareness or daily occupancy. However, the proposed model separates daily human behaviour of occupants (which is based on social parameters) from their energy awareness. More details will follow in Section 4.

\section{Background: Behaviour Change Theories and Energy Interventions}

\subsection{Behaviour Change Theories}

Humans beings can be highly affected by the behaviour of others. Based on this observation, the theory of collective behaviour was formalised in Granovetter's threshold model [5] to explain the diffusion of a behaviour due to social contagion. The model follows a simple decision rule, where individuals choose to adopt a behaviour when the percentage of others doing the behaviour exceeds a threshold. This threshold represents a complex combination of norms, values, motives, beliefs, etc. Once the threshold is exceeded, it is considered that the net benefit of the behaviour exceeds the perceived costs. The threshold model has been widely used in several applications such as effective targets to influence collective behaviour [12]. The other human behaviour theory used in this model is cognitive dissonance by Festinger [6]. Dissonance is defined as the inconsistency that happens between the individual's knowledge, opinion, beliefs, or attitudes, which are the cognitive factors that drive behaviour. Based on the fact that dissonance is uncomfortable, Festinger [6] proves that humans try to reduce it by adapting their behaviour or changing one or more of the cognitive factors. One of the major sources of dissonance are social groups. Therefore, observing others doing a behaviour that is very different from the individual's behaviour or spreading a general belief that a specific behaviour is not accepted, drives members of a social group to adapt their behaviour, thus reducing the uncomfortable dissonance. Besides, as the magnitude of dissonance increases, it is expected that the tendency to reduce it will increase. The magnitude of dissonance is affected by (1) the number of others who hold a different behaviour, and (2) the level of difference between the individuals' behaviours.

\subsection{Energy Efficiency Interventions and Peer Pressure}

Given the high percentage of energy consumption in residential buildings, research and policy makers efforts have been focused on promoting energy efficient behaviour, technologies, and structural improvements. This paper is focused on the behavioural aspect by modelling energy efficiency interventions. The target of interventions is to motivate occupants to adopt energy efficiency behaviour by working on their values, attitudes, beliefs, and knowledge [13]. Interventions can be of many forms such as goal setting, information (workshops, mass media campaigns, and home audits), rewards, and feedback [13]. In many occasions, these interventions take advantage of the peer pressure effect by comparing ones behaviour with the behaviour of others. Peer pressure is the influence that members of the same community have on each other which leads to change in behaviour. This effect is shown to be the most influential reason of environmental behaviour 
change [4]. This is because information received from personal relationships are better recognised and remembered than other sources of information [14].

\section{Methodology}

\subsection{The Agent-Based Model}

The proposed family pressure model is based on the ABM developed in Abdallah et al $[15,16]$. The model simulates energy consumption behaviour of families. Every occupant is represented by an agent that acts in a house environment and interacts with appliances. The inputs of the model are the social parameters including family size, ages, and employment types (full/part-time job, unemployed, retired and school). Besides, the energy awareness type of occupants determines the probability of performing energy saving actions (e.g. turning off devices when not in use). This can be one of four types: 'Follower Green', 'Concerned Green', 'Regular Waster', and 'Disengaged Waster'. Each of these types is reflected in the model as a continuous attribute called 'energy awareness' between 0 and 100 based on a normal distribution as shown in the $2^{\text {nd }}$ and $3^{\text {rd }}$ column of Table 1 .

\begin{tabular}{c|c|c|c|c|c}
\multicolumn{6}{c}{ Table 1: Mean and Standard Deviation of Awareness Types } \\
\hline Awareness Type & Mean $\mu$ & $\begin{array}{c}\text { Standard } \\
\text { Deviation } \sigma\end{array}$ & Value $(a)$ & Abbreviation & Category \\
\hline Follower Green & 0.74 & 0.041 & 1 & $\mathrm{~F}$ & Green \\
Concerned Green & 0.72 & 0.043 & 2 & $\mathrm{C}$ & Green \\
Regular Waster & 0.41 & 0.033 & 3 & $\mathrm{R}$ & Waster \\
Disengaged Waster & 0.25 & 0.057 & 4 & $\mathrm{D}$ & Waster \\
\hline
\end{tabular}

The ABM is supported by probability distributions from an integrated probabilistic model based on large sets of real data. The distributions are used to generate realistic occupancy and activities based on the given social parameters. The simulation time is determined by the day of the week $(d)$ and 144 time steps per day $(t)$ each representing 10 minutes. During the simulation, the occupant agent selects an occupancy state $\left(o s_{t d}\right)$ which can be away, active at home, or sleeping, for a duration $(d r)$. The occupancy state is selected based on the occupant's previous state $o s_{(t-1) d}$, age, employment type $(e m p)$, day $(d)$, and time $(t)$ as shown in functions (1) and (2). When the occupant agent is active at home, it performs activities from the following set $\{$ Using the computer, Watching television, Listening to music, Taking shower, Preparing food, Vacuum cleaning, Ironing, Doing dishes, Doing laundry\}. The decision of doing an activity for a specific duration $(d r)$ depends on the occupant's age, employment type $(e m p)$, day $(d)$, and time $(t)$ as shown in function (3).

$$
\begin{gathered}
O S: \text { age }, e m p, o s_{(t-1) d}, t, d \rightarrow o s_{t d} \\
\text { age, emp,ostd }, t, d \rightarrow d r
\end{gathered}
$$

$A C:$ age $, e m p, t, d \rightarrow a c_{t d}, d r$

Every activity that the occupant performs is associated to an appliance $a$. Appliances are modelled as dummy agents that only react to occupant agents actions (turn $\mathrm{ON}$ and $\mathrm{OFF}$ ). When the occupant agent starts an activity, it turns the associated appliance ON. When the activity ends, it chooses to turn the appliance ON or OFF based on its energy awareness attribute (ea) and any 
other occupant $\left(O_{a}\right)$ who is sharing the same appliance according to functions 4 and 5 . For more details about the previous model, readers are referred to $[15,16]$.

$$
\begin{aligned}
T O_{a}: a c_{t d} & \rightarrow \text { turnOn }_{a} \\
& \quad a c_{t d}, O_{a}, e a \rightarrow\{k e e p O n, \text { turnOff }\}_{a}
\end{aligned}
$$

\subsection{The Family Pressure Model}

The family pressure model is composed of two sub-models: behaviour change sub-model, and energy efficiency interventions sub-model.

Behaviour Change Sub-Model The occupants behaviour change is motivated by Granovetter's threshold model [5] such that the occupant agents change their behaviour when a threshold is exceeded. Although Granovetter's model explains the effect of social pressure on behaviour, it does not fit to the family pressure effect on energy efficient behaviour for two reasons. First, the model is applied in a public community which has different values and motives, therefore different thresholds. However in a family setting, we consider that family members have similar values and motives based on the fact that they have chosen to live together or were raised together. Therefore, when adapting Granovetter's model to the application at hand, we consider one global threshold for the whole family. This does not revoke the fact that people react differently because we have set the global threshold as a probabilistic one [17] - so once the threshold is exceeded the individuals adopt the behaviour with a probability. Second, the threshold model considers binary decisions. However, energy consumption behaviour is a continuous behaviour that is performed at different levels. This difference led us to explore the well-established theory of cognitive dissonance by Festinger [6] which is used to adapt the threshold model to the energy consumption application. Based on the two factors that affect the magnitude of dissonance outlined in Section 3.1, we adapt the definition of the threshold to fit the energy consumption behaviour. The first factor goes along with Granovetter's threshold definition such that more adopters of a given behaviour leads to changing others' behaviour. The second factor is used to overcome the inapplicability of the threshold model with the energy efficiency behaviour being continuous. Therefore, we define the threshold as the difference between the individual's awareness type and the average of other's awareness types.

The time step in this model is set to 4 weeks of simulation time since individuals usually take time to observe the behaviour of others. In order to express awareness types in numerical values, every awareness type is given an integer value as shown in the $4^{\text {th }}$ column of table 1 . For a family composed of $N$ occupants, every time step $T$, each occupant agent $i$ calculates the difference diff $_{T i}$ between its awareness type $a_{i}$ and the average awareness types of others $a_{j}$, where $j \in[1, N]: j \neq i$ using equation (6).

$$
\operatorname{diff}_{T i}=a_{i}-\left(\sum_{j=1, j \neq i}^{N} a_{j}\right) /(N-1)
$$


Behaviour change happens if $\mid$ diff $_{T i} \mid$ exceeds the global threshold $d$ where $d \in$ $[0,4]$. A high threshold implies low sensitivity to cognitive dissonance and a low threshold implies high sensitivity to cognitive dissonance. The global threshold $d$ is a probabilistic threshold such that the occupant changes behaviour with probability $p$ where $p \in[0,1]$. This attribute is referred to as threshold lag [18] which explains the stochastic nature of human behaviour due to uncertainty and differences in the speed of reaction, where a higher value of $p$ means a higher rate of change. $p$ is set to 0.5 as a middle point between high and low rate of change throughout the simulations in this paper. Once behaviour change is decided, the awareness type of the occupant changes towards the average of other's awareness types assuming that the occupant is adapting her/his behaviour to be similar to others. Behaviour change is done by stepping between the awareness types one step at a time either to the green side (green effect) or the waster side (waster effect). The behaviour change process step is outlined in algorithm 1 which is repeated for every agent $i$ at every time step $T$.

\begin{tabular}{|c|c|}
\hline Algorithm 1: Behaviour Change Step & $\begin{array}{l}\text { Algorithm 2: Intervention Behaviour } \\
\text { Change Step }\end{array}$ \\
\hline $\begin{array}{l}\text { calculate diff } f_{T i} \text { using equation (6) } \\
\text { if } \mid \text { diff }_{T i} \mid \geq d \text { then } \\
\text { select random number rand } \\
\text { if rand } \leq p \text { then } \\
\qquad \begin{array}{c}\text { if diff } f_{T i}>0 \text { then } \\
\text { else } a_{i}=a_{i}-1 \\
\left\lfloor a_{i}=a_{i}+1\right.\end{array}\end{array}$ & 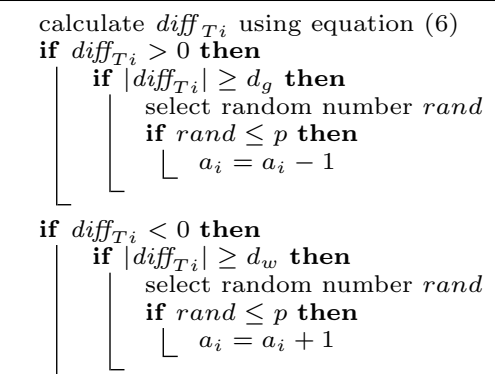 \\
\hline
\end{tabular}

Energy Efficiency Interventions Sub-Model This paper distinguishes between family-level interventions, and occupant-level interventions. Each of these interventions can be of any form as outlined in Section 3.2, but they differ in the number of occupants to target. The family-level intervention targets the family in general by changing its overall norms, values and beliefs. It can be applied by promoting the energy efficient behaviour such as giving financial incentives or repressing the wasting behaviour such as incurring charges [12]. The occupantlevel intervention targets the least aware occupant/s in the family and leads to increasing their awareness levels. These two types of interventions are considered to observe how the collective family pressure can help in achieving more aware occupants, thus less energy consumption. It also allows policy makers to decide the needed combination and intensity of interventions based on each family composition (in terms of awareness levels and social parameters).

When the family-level intervention happens, the overall norms, values and beliefs of the family change. The family-level intervention has two intensities which represent the efficiency or effort made to achieve better results. Therefore, $I_{p} \in[1,4]$ is defined as the promotion intensity and $I_{r} \in[1,4]$ as the repression intensity. These two types of family-level interventions are reflected 
by two thresholds: one that affects the promotion of green effect $d_{g} \in[0,4]$ and another that affects the repression of waster effect $d_{w} \in[0,4]$. Therefore, the intervention increases $d_{w}$ by $I_{r}$ thus increasing the cost to adopt waster behaviour and/or decreases $d_{g}$ by $I_{p}$ thus increasing the benefit of adopting the green behaviour as outlined in Granovetter [5]. $d_{g}$ and $d_{w}$ change in effect of the intervention based on equations (7) and (8) given the initial threshold $d$. For deciding behaviour change, $d_{g}$ is checked when there is a possibility to change towards the green side $\left(\right.$ diff $\left._{T i}>0\right)$, and $d_{w}$ is checked when there is a possibility to change towards the waster side $\left(\operatorname{diff}_{T i}<0\right)$ as shown in Algorithm 2. The occupant-level intervention does not change the threshold of the family because it targets specific occupants. It aims to change the awareness of occupants while the regular behaviour change step in Algorithm 1 is applied. The intervention can have an intensity $I_{o} \in[1,3]$ and can be applied to a member of the family $i$ at a specific time step $T$ according to equation (9).

$$
\begin{array}{rll}
d_{g}=d-I_{p} & : & d_{g} \in[0,4] \\
d_{w}=d+I_{r} & : & d_{w} \in[0,4] \\
a_{i(T+1)}=a_{i T}-I_{o} & : & a_{i T} \in[0,4]
\end{array}
$$

\section{Experiments and Discussion}

This section presents a number of experiments with different input parameters to show how varying these inputs can result in different intervention outcomes. It is worth to mention that this paper only presents a number of significant scenarios as a proof-of-concept while achieving the purpose of the paper. Abbreviations of awareness types ( $5^{\text {th }}$ column of Table 1 ) are used to identify the initial awareness of the family, such that a four occupant family with one 'Follower Green' and three 'Disengaged Wasters' is denoted by FDDD. In every simulation run, 100 households were simulated to capture the stochastic effect of the threshold lag ${ }^{1}$. The scenarios are run for a year and the resulting average yearly consumption and converged awareness types are recorded. These types were categorised based on the number of Green occupants in the family (represented in the figures by different colours in the bars). The categories of the awareness types are determined by the last column of Table 1 .

\subsection{Family Pressure Convergence}

The aim of this experiment is to observe the resulting awareness types as an effect of family pressure based on different thresholds. Figure 1 shows the results of three scenarios: (a)FFFD, (b)FCRD, and (c)FDDD. The last scenario of every bar graph $(\mathrm{d}=4)$ shows the initial category of the family because $\operatorname{diff}_{T i}$ can be maximum 3, thus no change in awareness types.

In scenario (b), the family remained with two green occupants at thresholds 2 and 3 , besides, in (a) and (c) the family remained the same at threshold 3 and changed only one occupant at threshold 2 . This indicates that the family does

\footnotetext{
1 The model was validated by running a number of scenarios with different random numbers seed where the results came out to be similar
} 


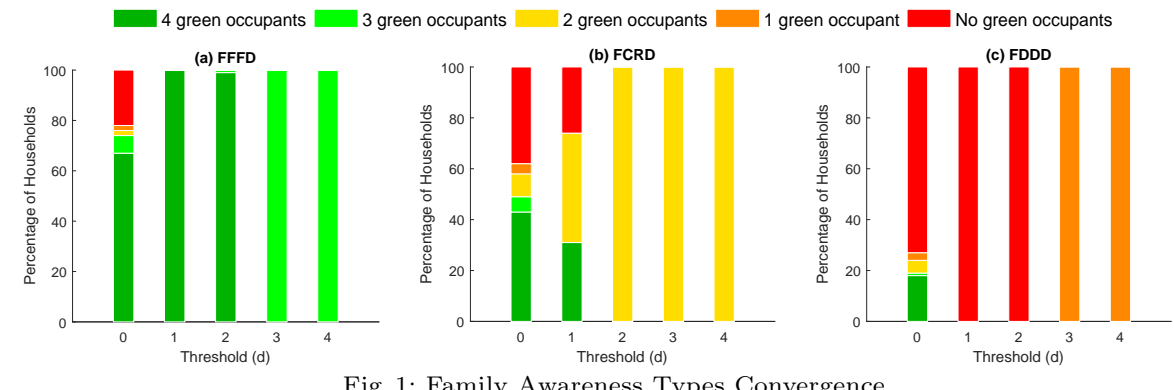

not change significantly when the threshold is high $(\mathrm{d}=2$ and 3$)$. However, at low thresholds ( $\mathrm{d}=0$ and 1 ), the family converged mainly towards the dominant awareness type. For example, in (a) the convergence was mostly towards ' 4 green occupants', because initially there were three green occupants. A similar observation was noticed in (c). In scenario (b) where there is no dominant awareness type, the convergence was with equal probabilities either to all green occupants or all waster occupants ('no green occupants' category) with higher convergence to the extremes at threshold 0 . These results indicate that the proposed model reflects the theory of cognitive dissonance and collective behaviour which agree that people tend to change their behaviour to conform with the behaviour of others. It is worth noting that in (a) and at threshold 0 , around $20 \%$ of the households converged to 'no green occupants'. This means that the only waster occupant succeeded to change the behaviour of the other three green occupants. This phenomenon is explained in the cognitive dissonance theory which states that dissonance can be reduced by either adapting with others, or convincing the others to adapt with the individual. This explains how the three green occupants converged to wasters in effect of one waster occupant as in (a) and vice versa in (c). Festinger [6] mentions that in this case, the overall cognitive elements of the surrounding environment change, but this is easy when the individual can find others who hold the same behaviour, which explains the low percentage of this convergence ( $20 \%$ in our experiment).

\subsection{Family-level Intervention}

In this experiment, family-level interventions are applied to scenario (c) of the experiment 1 (FDDD) as it has the most waster occupants after convergence. For each threshold, the possible intensities of family-level interventions are applied keeping the thresholds $d_{g}$ and $d_{w}$ in their limits $[0,4]$. The aim of this experiment is to show the effect of promotion and repression interventions when varying their intensities. Figure 2 shows the results with initial thresholds 0,1 and 2 .

It is noticed at thresholds 1 and 2 that the number of green occupants increases as the promotion intensity $\left(I_{p}\right)$ increases, which is not the case with repression intensity $\left(I_{r}\right)$ where most of the occupants stayed wasters. This indicates that repression intervention is less efficient than the promotion intervention. This is attributed to the high number of waster occupants, such that encouraging them to adopt the green behaviour is more effective than repressing the only green occupant from getting affected by waster occupants. Another 


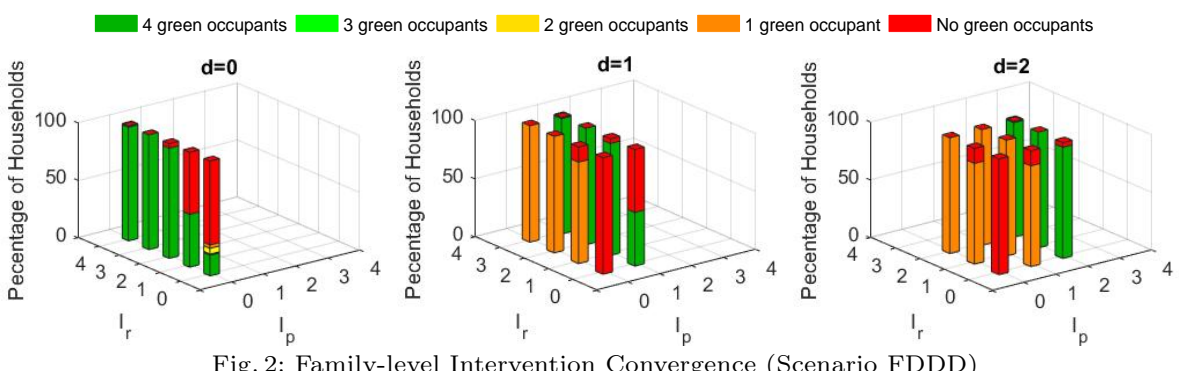

indication from varying intervention intensities is inferring the minimum intensity needed to increase the possibility of getting 4 green occupants. For example at threshold 0 , repression intensity 2 is enough to get ' 4 green occupants' with probability more than 0.95 . This allows to identify the minimum effort needed while achieving the maximum number of green occupants.

\subsection{Occupant-level Intervention}

This experiment studies the effect of occupant-level interventions which directly change the awareness of least aware occupants. Scenario FFFD with threshold 0 is selected to get the minimum intensity required to prevent the 'no green occupants' convergence (as shown in scenario (a) in section 5.1). As the family initially has one waster occupant, the intervention is applied for one occupant with different intensities. Besides, the intervention can be applied at specific times of the year, therefore it can be an 'early intervention' at $T=2$, 'mid-year intervention' at $T=6$ or 'late intervention' at $T=9$. This determines the best intervention time just before the waster occupant affects other green occupants. Figure 3 shows the results while varying the intervention time and intensity.

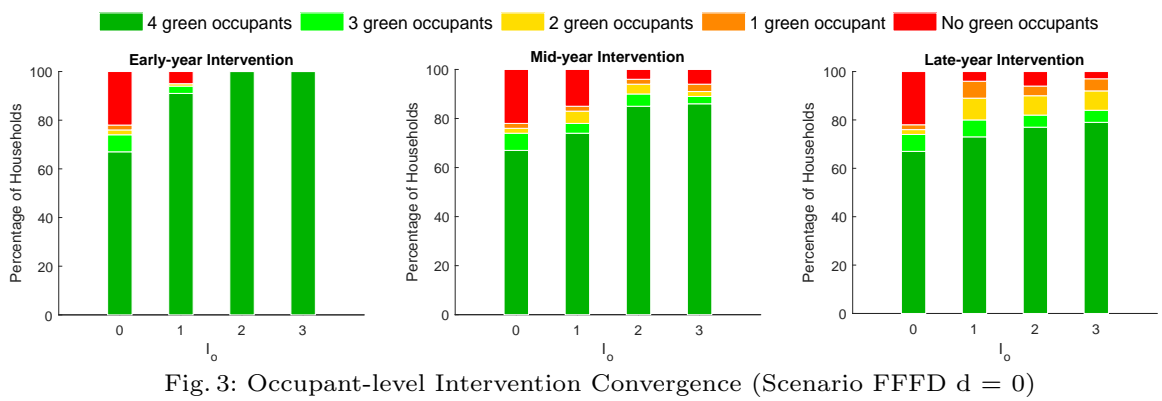

It is observed that as earlier the intervention and as higher its intensity, as more green occupants are obtained. The early interventions with intensities 2 and 3 are the most effective with no waster occupants after a year. This is expected because the waster occupant is affected by the external intervention at an early stage, thus leading to 4 green occupants. However, in all other scenarios, waster occupants are observed even at higher intensities. This shows that one intervention per year is not enough to make an impact on families with only one waster occupant. This suggests to perform continuous interventions to maintain the green effect and combine them with family-level interventions. Note the this 
experiment was performed with very low threshold of the family $(d=0)$ so occupants can easily influence each other.

\subsection{Effect of Interventions on Families with Varied Social Parameters}

In our previous paper [16], it was concluded that social parameters affect the energy waste of the family. Although the previous model does not simulate family pressure, we showed that energy waste in large families is less than small families. On the basis of this conclusion, the current experiment tests if a family-level intervention is more efficient in big families than small families. For this purpose, the family-level intervention is applied on (a) a two-occupant family and (b) a four-occupant family. Figures $4 \mathrm{a}$ and $4 \mathrm{~b}$ show the awareness types convergence of scenarios (a) and (b) respectively with an equivalent initial numbers of green and waster occupants (FD and FFDD) and threshold $d=0$. Figure $4 \mathrm{c}$ shows the resulting energy saving percentage when compared to the no-intervention scenario $\left(I_{r}=0\right)$ and the convergence time which is the time it takes the family to reach a stable state where the occupants are no more affected by each other.
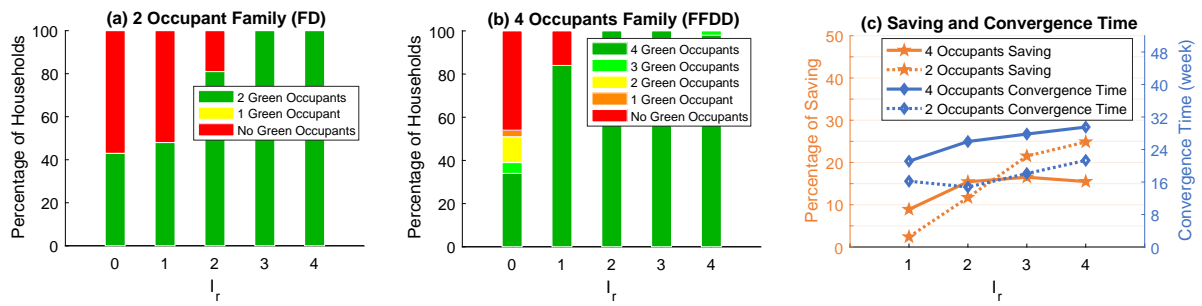

Fig. 4: Effect of Family-level Intervention on Two and Four Occupant Families $(\mathrm{d}=0)$

In Figure $4 \mathrm{c}$ at intervention intensities 1 and 2, the percentages of saving for big families are $9 \%$ and $16 \%$ respectively, which are more than that of small families (i.e. $1 \%$ and $11 \%$ ). This is also observed in the awareness types convergence (Figures $4 \mathrm{a}$ and $4 \mathrm{~b}$ ) where the ' 4 green occupants' category is more dominant in (a) than the ' 2 green occupants' category in (b). However, at intensities 3 and 4 , the savings of small families are $21 \%$ and $25 \%$ respectively, which dominates that of big families (i.e. 16\% and 15\%)(Figure 4c). Besides, all of the occupants in scenarios (a) and (b) converged to green occupants as shown in Figures 4a and $4 \mathrm{~b}$. This is explained by the lower convergence time of small families (figure 4c). This means that a higher intensity intervention converges small families quicker than big families which consequently leads to higher saving. Thus, the family-level intervention can result in maximum saving at low intensity in big families as opposed to small families. While a high intensity intervention is more efficient in small families as it leads to a larger and quicker saving than big families. This experiment can be repeated with varied social parameters, thresholds, and intervention types to obtain the most efficient intervention in every case.

\subsection{Discussion}

The model proposed in this paper simulates peer pressure effect on energy awareness levels and consumption of families. The peer effect behaviour of occupants 
is based on two human behaviour theories opposed to other models that do not use existing theories [9]. The behaviour theories were adapted to comply with the energy consumption behaviour and family environment, while other models use different theories that simulate office environments [11]. Beside, the current model offers different options of input including social parameters (family size, employment types, ages), awareness levels, values and beliefs that affect the energy consumption behaviour, and intervention options. We proved in the experiments that these inputs affect the outcome of interventions. The experiments focused on demonstrating the application of the model in pre-specified scenarios. The model can ideally be used to study the impact of any intervention planned by governing bodies on the outcome (i.e. energy saving). This can be done by estimating unknown parameters, running the model with initial parametrisation of known and unknown parameters. Then a search mechanism (e.g. grid search) is applied to best estimate the unknown parameters, minimising the difference between the model's synthesised data and the observed real data. If the search space is large, in case of having too many unknown parameters, computational intelligence methods like Genetic Algorithm can be applied. Revealing these unknown parameters can help in determining the reason why interventions are effective in some cases, but not in others.

\section{Conclusion and Future Work}

This paper presented an ABM that simulates energy awareness peer pressure in a family setting. The model uses the collective behaviour theory and the theory of cognitive dissonance to reflect realistic peer effect. Different energy efficiency interventions can be applied and the resulting awareness types and savings are observed. The presented experiments show that the human behaviour theories are well-reflected in the model. Besides, they show how the model can offer an analytical tool for governing bodies to analyse the effect of interventions and make decisions of how to target different families to get the best results.

A variation of this model is to make the effect of members depend on how often they are in contact in the house, which makes the interaction more realistic. This can be easily achieved because the ABM simulates individuals' daily availability at home in a 10-minute time step. The current model have not considered a weighting attribute which determines the level of relation between the occupants which affects the level of influence. This attribute can be added in the future where the intervention may be targeted at a specific relationship if it proves efficient. Also the modelling of behaviour change can be done at the energy awareness level, not at the awareness type level. This can enhance model's capability to simulate more fine-grained behaviour change. These enhancements are expected to produce an even more realistic model that reflects the quality and rate of daily interactions among the family members.

\section{References}

1. US Global Cilmate Change Research Program (USGCRP): Global Climate Change. In: Global Climate Change Impacts in the United States. Cambridge University Press, New York (2009) 13-26 
2. Internation Energy Agency (IEA): Electricity Information Overview 2017. Technical report, IEA (2017)

3. Zipperer, A., Aloise-Young, P.A., Suryanarayanan, S., Roche, R., Earle, L., Christensen, D., Bauleo, P., Zimmerle, D.: Electric Energy Management in the Smart Home: Perspectives on Enabling Technologies and Consumer Behavior. Proceedings of the IEEE 101(11) (2013) 2397-2408

4. Nolan, J.M., Schultz, P.W., Cialdini, R.B., Goldstein, N.J., Griskevicius, V.: Normative Social Influence is Underestimated. Personality and social psychology bulletin 34(7) (2008) 913-923

5. Granovetter, M.: Threshold Models of Collective Behaviour. American journal of sociology 83(6) (1978) 1420-1443

6. Festinger, L.: A Theory of Cognitive Dissonance. Vol. 2 edn. Stanford University Press, Stanford, California (1962)

7. Epstein, J.M.: Agent-Based Computational Models and Generative Social Science. Complexity 4(5) (1999) 41-60

8. Axtell, R.: Why agents? on the varied motivations for agent computing in the social sciences. Technical report, Center on Social and Economics Dynamics: The Brookings Institution (2000)

9. Azar, E., Menassa, C.C.: Agent-Based Modeling of Occupants and Their Impact on Energy Use in Commercial Buildings. Journal of Computing in Civil Engineering 26(4) (2012) 506-518

10. Chen, J., Taylor, J.E., Wei, H.H.: Modeling building occupant network energy consumption decision-making: The interplay between network structure and conservation. Energy and Buildings 47 (2012)

11. Azar, E., Menassa, C.C.: A comprehensive framework to quantify energy savings potential from improved operations of commercial building stocks. Energy Policy 67 (2014) 459-472

12. Hu, H.H., Lin, J., Cui, W.T.: Intervention strategies and the diffusion of collective behavior. Journal of Artificial Societies and Social Simulation 18(3) (2015) Paper 16

13. Abrahamse, W., Steg, L., Vlek, C., Rothengatter, T.: A review of intervention studies aimed at household energy conservation. Journal of Environmental Psychology 25(3) (2005) 273-291

14. Costanzo, M., Archer, D., Aronson, E., Pettigrew, T.: Energy Conservation Behavior. The Difficult Path From Information to Action. American Psychologist 41(5) (1986) 521-528

15. Abdallah, F., Basurra, S., Gaber, M.M.: A hybrid agent-based and probabilistic model for fine-grained behavioural energy waste simulation. In: IEEE 29th International Conference on Tools with Artificial Intelligence (ICTAI), IEEE (2017) 991-995

16. Abdallah, F., Basurra, S., Gaber, M.M.: Cascading probability distributions in agent-based models: An application to behavioural energy wastage. In: 17th International Conference on Artificial Intelligence and Soft Computing (ICAISC), Springer (2018)

17. Kiesling, E., Günther, M., Stummer, C., Wakolbinger, L.M.: Agent-based simulation of innovation diffusion: A review. Central European Journal of Operations Research 20(2) (2012) 183-230

18. Bohlmann, J.D., Calantone, R.J., Zhao, M.: The Effects of Market Network Heterogeneity on Innovation Diffusion: An Agent-Based Modeling Approach. Journal of Product Innovation Management 27(5) (2010) 741-760 